\title{
BIM EDUCAÇÃO, UMA REVISÃO BIBLIOGRÁFICA ESTRUTURADA
}

\author{
Juliana Mizumoto $^{(1)}$, Francisco Oliveira ${ }^{(1)}$ \\ (1) CIAUD, Faculdade de Arquitetura, Universidade de Lisboa, Lisboa, Portugal
}

\begin{abstract}
Resumo $^{1}$
A utilização da metodologia Building Information Modeling (BIM) é uma realidade na era da Construção 4.0, a digitalização dos processos torna-se necessária e deste modo também a utilização de ferramentas e novos conceitos de gestão de projetos. Nessa conjuntura, a formação de novos profissionais para suprir o novo mercado de trabalho revela-se ser um desafio. Este desafio também se coloca às Universidades, que ainda não possuem uma abordagem clara sobre o assunto. Verifica-se que existe hoje um incremento de publicações sobre o tema e este artigo apresenta uma abordagem estruturada ao problema através de uma análise bibliométrica com foco nas publicações existentes sobre o tema BIM Educação.

A metodologia adotada passou pela seleção de publicações existentes, através da plataforma de pesquisa Web of Science e posterior tratamento, com a utilização do software VOS Viewer para análise dos dados e respetiva transformação em imagens baseadas nas teorias de visualização do conhecimento, a partir do conceito de mapeamento da ciência (science mapping).

Essa pesquisa faz parte de um projeto de investigação para tese de doutoramento e neste artigo serão expostos os resultados de uma fase da investigação, a qual teve como objetivos: o mapeamento do conhecimento relacionado com o tema; demostrar os tópicos abordados mais relevantes, visualizar geograficamente os pontos de maior relevância no assunto; além de identificar as relações entre as publicações e autores.

Esta investigação pretende contribuir para discussão de um caminho possível para a organização de uma metodologia de ensino do BIM no contexto universitário e explicita uma metodologia de trabalho para a análise de dados bibliográficos.
\end{abstract}

\footnotetext{
${ }^{1}$ Este texto faz parte da pesquisa realizada pela primeira autora, no âmbito do Curso de Doutorado em Arquitetura pela Faculdade de Arquitetura da Universidade de Lisboa, sob a supervisão do segundo autor. O trabalho de pesquisa é realizado no contexto da tese de doutorado em andamento, intitulada. "BIM Manager: Um novo escopo de trabalho para Arquitetos na Coordenação de Projetos da Construção Civil”.
} 


\section{Introduçãa}

Integrar o ensino BIM no currículo das universidades de arquitetura e engenharia é um fator crucial para a disseminação da metodologia, já que a falta de profissionais capacitados é provavelmente a maior barreira para a disseminação do uso desta tecnologia na indústria da construção [1]. Este é um movimento que tem vindo a ocorrer progressivamente e as universidades têm-se adaptado, mas verifica-se que são múltiplos os caminhos seguidos para atender à esta necessidade, aplicados em realidades distintas e, consequentemente, não se consegue encontrar uma uniformidade nas formas ou conteúdos tratados. Procurando compreender o panorama BIM Educação atual, esta pesquisa apresenta os primeiros resultados de uma investigação estruturada sobre o tema, isso se dá a partir dos produtos das buscas sobre a estrutura científica desta especialidade, isto é: o conjunto mais importante de documentos sobre o tema e respetivas relações cruzadas.

A estrutura deste documento organiza-se da seguinte forma: (i) argumentação para utilização do método da análise bibliométrica através da literatura específica, (ii)utilização do software VOS Viewer, (iii)mapas de resultados.

\section{Bibliometria}

O principal método para organização desta investigação é a análise bibliométrica. Há diversas definições para o termo na literatura específica [2] e este pode ser traduzido como sendo o trabalho de análise quantitativa e estatística de livros, artigos e outras publicações, que permite monitorizar e analisar a estrutura de crescimento da ciência [3].

O método quantitativo para analisar dados da bibliografia, pode orientar o investigador mesmo antes do início de uma investigação, pois do resultado analítico preliminar é possível visualizar o panorama do complexo campo de assuntos a serem estudados [4]. Tal cenário configura um extenso sistema, formado por relações sociais entre pesquisadores, suas bases de conhecimento e arquivos de publicações [5]. A bibliometria ainda ameniza o eventual enviesamento subjetivo que pode ocorrer por parte do investigador durante o processo de pesquisa bibliográfica de referência, já que com esse método, podemos ter acesso a dados bem mais orientados e recorrendo a um maior e mais variado número de juízos emitidos por pesquisadores que trabalham no mesmo campo de investigação.

Quantificar a literatura e as suas relações é um processo já estudado por diversos especialistas [6], [7] destacando-se o trabalho de Garfield, que defende a ideia de que uma referência altamente citada indica que um grande número de pesquisadores encontrou utilidade para o tema, logo, o fator de importância ou impacto da publicação aponta que esta é cientificamente útil [7]. Impacto esse que é utilizado na avaliação e tomada de decisões por parte das Universidades, entidades governamentais e agências de financiamento [3], as quais utilizam ferramentas, índex, parâmetros de comparações, reconhecimento de influências e eficiência, refletindo-se isto em maior ou menor investimento em determinadas linhas de pesquisa ou Instituições de ensino. Em consequência destes factos, a visualização e compreensão dessa rede de relações bibliográficas torna-se estimulante para os profissionais da área [8]. 


\section{Mapeamento Gráfico}

Os mapas são a representação dos resultados da bibliometria, e no contexto da ciência, representam o modo como as disciplinas, especialidades, artigos individuais ou autores estão corelacionados entre si [8], com objetivo de identificar os seus conceitos, teorias e modelos, agrupamentos de ideias que formam a base do conhecimento, as fronteiras e influências cruzadas entre os investigadores [5]. A importância de ilustrar as relações entre pesquisas está descrita na literatura científica [9], verificando-se que há situações em que algumas publicações expõem-se afastadas, mas possuem o mesmo propósito, enfatizando a importância para a ciência que podem ter as interações temáticas não intencionais, que permitem o surgimento de novos "insights" no atual estado do conhecimento [8].

Há diferentes tipos de dados passíveis de serem extraídos da análise dos dados sobre as publicações, dentre eles estão as redes de relações de citações entre publicações, co-autoria, locais de publicações ou relações de co-ocorrência entre palavras chave [10]. A análise desses dados tonou-se uma atividade complexa devido à grande quantidade de informação, a simples experiência e conhecimento individual do pesquisador tornaram-se insuficientes para dominar a amplitude da produção científica atual. Em consequência, a preocupação no desenvolvimento de métodos para tratamento de dados [6] e visualização gráfica facilitada [11] tornou-se uma abordagem poderosa de análise das bases bibliográficas. Um dos passos dados mais relevantes para a ciência foi proposto por Garfield [12], quando deu início ao desenvolvimento do Sience Citation Index(SCI), que foi a base para as teorias de análise de citação atuais [13]. Contudo, foi somente a partir da disponibilização on-line de bases de dados que ocorreu a disseminação e proliferação de estudos que utilizam este método [4].

\section{Visualização}

Há diversas técnicas para o mapeamento e visualização dos domínios da ciência, como demonstra Borner [13], e estudos sobre visualização das redes de bibliometria, [4], [10] expõem os principais tipos de relações. Morris [5] demonstrou-nos as relações de forma estruturada e é uma das bases utilizadas nesta investigação.

Um dos primeiros aliados na facilitação das análises foi o programa de mapeamento SCI-Map [14], que demonstrava o processo de agrupamento (clusters), permitia ao pesquisador controlar e visualizar os dados em imagem $2 \mathrm{~d}$, por meio de um processo de triangulação dos dados. A partir deste programa surgiram novas formas de análise de agrupamento, tornando-se cada vez mais automatizadas e sofisticadas acompanhando os avanços da computação. Tal evolução resultou numa melhoria da representação gráfica dos modelos através de pesquisas focadas nas questões técnicas [13], [15]. Visando uma satisfatória representação dos dados e empregando funcionalidades técnicas, foi introduzido em 2010 um novo programa: VOSviewer, o qual é utilizado nesta pesquisa como ferramenta. A escolha deste programa, em detrimento de outros, tais quais Pajek ou SPSS ocorreu em face da sua supremacia quer na qualidade da representação quer no rigor da análise de dados, baseado em estudos comparativos anteriores [10], [11], [16]. Compreender a estrutura das especialidades é um caminho para mensurar a ciência, que não tem como função apenas produzir conhecimento público, mas também produzir conhecimento validado [5]. Validação esta que é possível de fazer através da aglomeração dos resultados em nuvens de similaridade (clusters), formando um modelo que representa a estrutura das 
especialidades e evidência as relações de interconexão entre elementos. A visualização explorada nesta investigação é a suportada pelo VOSviewer, baseada em distância entre os grupos de dados, técnica debatida por Waltman, van Eck e Noyons [17].

\section{Análise bibliométrica aplicada ao tema BIM Educação: VOSViewer}

A aplicação da análise bibliométrica nesta investigação iniciou-se pela busca sistemática e estruturada através de uma matriz de palavras chave: 1-BIM, 2-Building Information Modeling, 3-Building Information Modelling, 4-Building Information Model; a-Educational, b-Education, c-Teaching, d-Learning, e-Course, f-Curriculum, d-Training; e-Construction education, fArchitectural education. As combinações foram aplicadas na plataforma Web of Science, num período de busca suportado pelo software: de 1900 a 2019 (ano de execução da busca), resultando em 670 publicações trabalhadas através dos filtros de interesse do programa.

Parâmetros foram definidos para iniciar as análises e a escolha visou encontrar um panorama geral do ambiente científico abordado, fundamentado por critérios utilizados em estudos anteriores [13]. Para analisar as bases de dados é recomendado pela literatura especializada [4] a organização em grupos de interesse de resultados, assim como um planeamento prévio das atividades. Seguindo essa recomendação, os objetivos das análises foram ordenados em três grupos: (1) Estrutura da rede de conhecimento (grupos de publicações, autores e conceitos), (2) a dinâmica das relações durante o período das publicações registadas e (3) nas questões da investigação. Os resultados aqui discutidos são referentes à Estrutura de conhecimento do tema BIM Educação.

\section{Estrutura da rede de conhecimento: Temas}

A primeira procura de relações do tema BIM Educação foi feita pela co-ocorrência de palavras chave com objetivo de criar uma estrutura homogénea [5], e então encontrar os conceitos e assuntos mais abordados pelos especialistas. Os parâmetros utilizados foram:1-co-occurence, 2-all keywords, 3-full counting. Esse teste gerou uma lista de dados posteriormente tratados, e as suas duplicidades foram excluídas. Essa análise resultou em 1833 palavras chave relacionadas, e 84 delas dentro das fronteiras estabelecidas pelos filtros.

O resultado deste filtro (Figura 1Erro! A origem da referência não foi encontrada.) apresenta os assuntos publicados de maiores interesse por parte dos especialistas, e interpreta-se, por via deste método que quanto maior são as relações entre os dados, maior é a proximidade entre eles [10]. Sendo assim, os dados no centro têm mais inter-relação que os das extremidades, os quais são interpretados como assuntos menos correlacionados, até mesmo, em desenvolvimento, ou como novidade na especialidade.

A partir da análise dessa rede de relações, criam-se três níveis de hierarquia, o que orienta e direciona futuras buscas sobre o tema: $1^{\circ}$ nível: BIM Educação, $2^{\circ}$ nível - grupos de maior interesse, $3^{\circ}$ nível: subtemas. Dos resultados, extraem-se 8 grupos de temas que se relacionam em maior intensidade com assuntos mais específicos. Assim, pela métrica de peso de relações aplicada pelo VOSViewer, pode-se dividir o $2^{\circ}$ nível em: 1- Building Information Modeling, 2BIM, 3-Design, 4-Implementation, 5-Technology, 6-Information, 7-Construction, 8- 
Methodology. O $3^{\circ}$ nível organiza os subtemas relacionados, gerando novas possibilidades de abordagem para aprofundamento das especialidades encontradas (Tabela 1).

Essa classificação representa os resultados para as palavras que mais se relacionam e não necessariamente que estejam estáticas nas suas relações com outras e demais grupos (Figuras 4, 5 e 7), assim sendo, o resultado é um indicador do modo como a rede de conhecimento do BIM Educação se tem estruturado e dela podemos partir para outras análises.

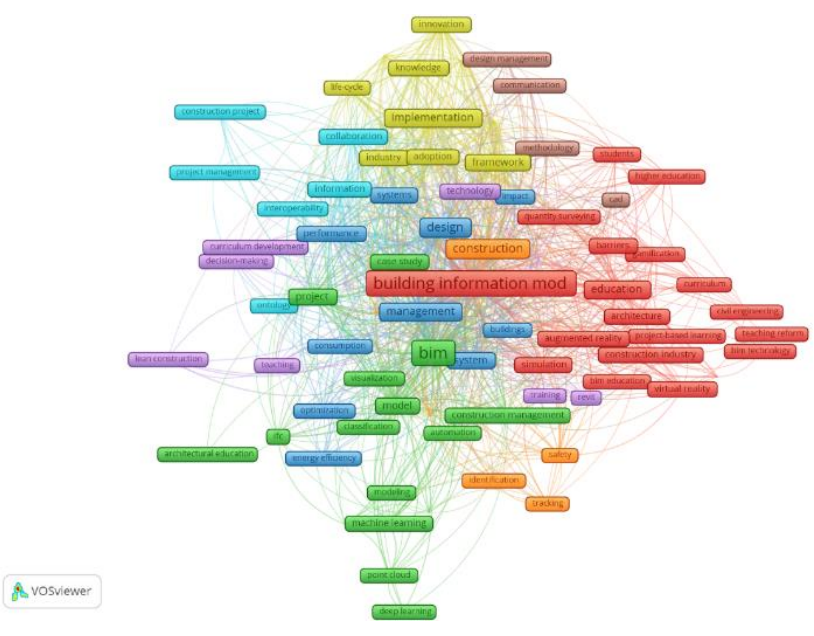

Figura 11: Estrutura dos temas.

Tabela1: Temas divididos em níveis

\begin{tabular}{|c|c|c|}
\hline \multicolumn{3}{|r|}{ Nível 1: BIM Educação } \\
\hline Grupos & Nível 2 & Nível 3 \\
\hline 1 & $\begin{array}{l}\text { building } \\
\text { information } \\
\text { modeling }\end{array}$ & $\begin{array}{l}\text { education, simulation, augmented reality, architecture, barriers, } \\
\text { construction industry, students, sustainability, gamification, virtual } \\
\text { reality, higher education, curriculum, quantity, surveying, civil } \\
\text { engineering, engineering education, project-based learning, BIM } \\
\text { education, BIM technology, teaching reform }\end{array}$ \\
\hline 2 & BIM & $\begin{array}{l}\text { model, project, construction management, visualization, IFC, } \\
\text { automation, case study, machine learning, classification, } \\
\text { photogrammetry, modeling, reconstruction, point cloud, laser } \\
\text { scanning, deep learning, architectural education }\end{array}$ \\
\hline 3 & design & $\begin{array}{l}\text { management, system, performance, systems, impact, buildings, } \\
\text { optimization, consumption, prefabrication, energy efficiency }\end{array}$ \\
\hline 4 & implementation & $\begin{array}{l}\text { framework, challenges, adoption, industry, innovation, integration, } \\
\text { knowledge, life-cycle, construction projects }\end{array}$ \\
\hline 5 & technology & $\begin{array}{l}\text { university, construction education, teaching, decision-making, Revit, } \\
\text { training, curriculum development, lean construction }\end{array}$ \\
\hline 6 & information & $\begin{array}{l}\text { collaboration, cost estimation, interoperability, ontology, construction } \\
\text { project, project, management }\end{array}$ \\
\hline 7 & construction & facilities management, safety, requirements, identification, tracking \\
\hline 8 & methodology & design management, communication, cad \\
\hline
\end{tabular}


A partir dos resultados dos níveis de relações, foram criados mapas para a visualização dos oito subtemas, o parâmetro utilizado foi a hierarquia gerada pelo programa no item com maior fator de relação do grupo analisado. Para este trabalho, foram selecionados três mapas que exemplificam a metodologia desenvolvida. O primeiro deles, "Education" (Figura 2-a) apresenta uma rede esparsa e diversa, demonstrando a gama alargada de assuntos diversos que têm sido estudados pelos especialistas para os relacionar com o tema central. Como exemplos: "curriculum", o investimento na criação e testes de métodos de estudo BIM, com integração de ferramentas que envolvam melhoria no ensino da metodologia nas universidades [18], e na preocupação em encontrar, desenvolver as competências exigidas pela indústria [19]. O tema "virtual reallity" está relacionado também com a educação BIM por referências de casos de estudo que aplicam a realidade virtual por meio da utilização de plataforma de colaboração [20], ou da criação de protótipos de modelo para serem utilizados na avaliação implementação BIM e do nível de conhecimento dos alunos através do novo método de ensino [21]. Outro tema relacionado é o "framework", podendo ser ilustrado pelo interesse crescente nesse formato de organização da informação para ser utilizadas de maneiras diversas, tais como: criação de um ambiente comum sobre cultura BIM global para se unificar conceitos e aplicá-los de forma multidimensional [22], na criação de frameworks dos assuntos que precisam ser ensinados e mesclados ao programa existente [23], ou na criação de um método pedagógico novo que considere as novas competências, nível de integração necessário em cada disciplina [24].

Enquanto resultado expectável para o mapa de framework, observa-se uma significativa relação com as palavras chave-centrais, mas também muitas outras relações de dimensões equivalentes com outros grupos. Pode afirmar-se que existe uma relação com os oito subtemas encontrados no mapa geral. Esse resultado argumenta a favor da importância dada pela ciência ao ensino de áreas que abrangem diversos usos da metodologia BIM.

Para o subtema "Design" ( $3^{\circ}$ grupo), a palavra de relevância para a investigação é a "Management" (Figura 2-b), e demonstra a importância do tema gestão no âmbito da metodologia BIM. Este era um resultado previsível, visto que é um assunto intrínseco à utilização do BIM em qualquer nível de aplicação e a gestão de projetos. É uma resposta que fortalece a validação dessa estrutura criada como método, e pode ser ilustrado com caso de estudo que aplica ferramentas de colaboração e gestão para teste de modelos federados [25], situação que depende e proporciona desenvolvimento das habilidades também relacionadas à gestão.

Representando o subgrupo "Technology" (5" grupo), o tema "University" apresentou resultados aparentemente dispersos, porém, revela relação entre os temas ligados ao ensino de software, metodologia, projeto. Neste mesmo grupo foram também investigados os resultados para o mapa "Construction Education" (Figura 2-c). A relação de interesse entre os assuntos demonstra uma orientação da engenharia para conteúdos da indústria, tecnologia, gestão, além de uma nova, mas não menos importante relação com realidade virtual, gamificação, realidade aumentada, visualização e segurança. Uma interpretação possível é a importância dos novos usos BIM na indústria, ilustrados pela utilização de mapeamento automático de edifícios por nuvem de pontos pra aplicação em gestão de operação e manutenção [26], ou pela automação de edifícios com aplicação de eficiência energética [27]. Portanto, fazem parte da estrutura de conhecimento da especialidade relacionada também ao ensino. 


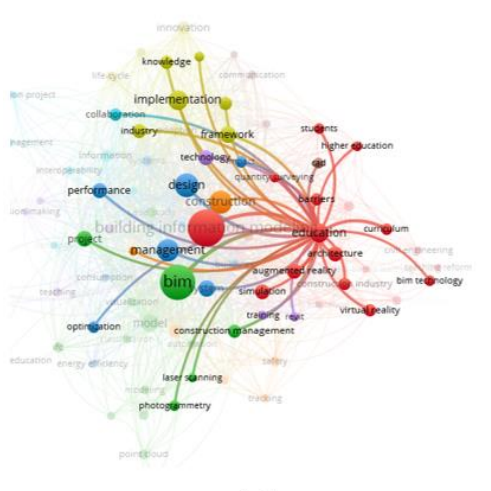

(a)

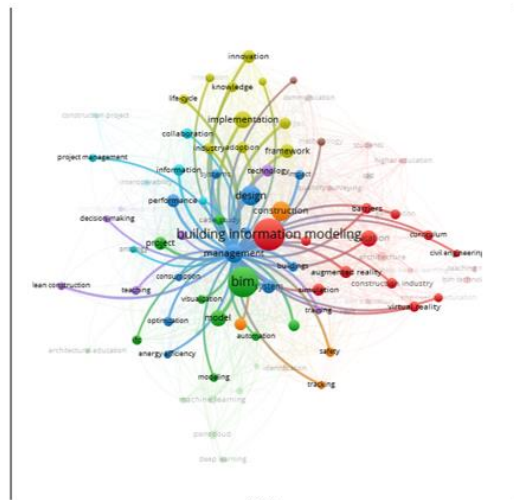

(b)

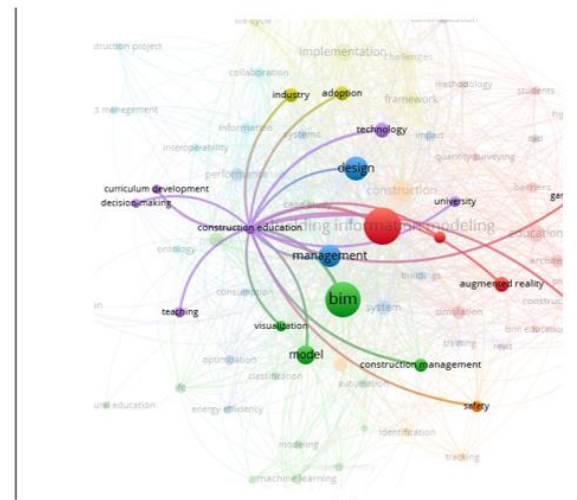

(c)

Figura 12: (a)Education, (b) Management, (c) Construction education.

\section{Estrutura da rede de conhecimento: locais}

Conhecer a localização geográfica relacionada aos documentos de maior relevância de um tema é uma forma de mapear a ciência. Seguindo a mesma base de dados inicial, foram criados mapas para identificar: (i)os países de maior relevância no tema BIM Educação, (ii) as Instituições de ensino às quais representam os pesquisadores e (iii) os canais de publicação mais utilizados.

O mapa de relações entre países mostra quarenta e oito elementos (Figura 5), com a liderança dos EUA (weight citation 527), seguido pela China (weight citation 322). Já a análise das Instituições mostra 163 elementos relacionados, os quais dividem-se em 18 grupos e hierarquizados pelo peso de número de citações exposto pelo programa. A tabela 2 é o resumo de 10 desses grupos, onde foram consideradas as Instituições mais relevantes de cada um deles. O mesmo raciocínio foi aplicado para a busca dos Journals mais relevantes para o tema, onde 74 itens foram selecionados e divididos em 18 grupos, resumidos na Tabela 3.

\section{Discussão}

Conforme exposto, este trabalho faz parte de uma investigação estruturada sobre o BIM Educação, os dados aqui expostos partem de um método de análise bibliométrica e exibem uma parte importante da estrutura do conhecimento dessa especialidade.

Conhecer a organização dos temas encontrados a partir das palavras-chave abre um caminho para uma estruturação pedagógica do BIM. A partir desse mapeamento de dados, algumas leituras podem ser feitas tais quais as expressas nesse trabalho, no sentido de direcionar o olhar científico para relações entre temas que podem não ser comummente trabalhados em conjunto, mas que na estrutura das publicações selecionadas, se mostram correlacionados. Outra perspetiva de leitura desses dados é sobre a divisão dos temas em grupos de interesses dos pesquisadores, ponto de referência para guiar um desenvolvimento do Estado da Arte sobre o assunto BIM Educação. 
Os dados de localização das publicações abrem caminho para uma análise sobre a polarização do conhecimento neste assunto, alertam a Academia sobre a necessidade de investimento na área do BIM Educação, além de uma maior colaboração entre as Instituições.

A partir desses dados, uma discussão que se coloca é a relação entre publicações relevantes vindas das Instituições citadas com a abordagem pedagógica que elas propõem em seus cursos. Essa é uma realidade importante de se compreender e abre caminho para o questionamento se, além de serem departamentos produtores de conteúdos relevantes, também produzem um ensino de BIM Educação de referência nos seus currículos.

Para além dos dados aqui expostos, outros foram coletados como forma de dar andamento às questões da Investigação principal que também aborda a discussão sobre a análise qualitativa dos documentos selecionados.

Com os dados da estrutura, é possível guiar novas pesquisas para se desenvolver modelos de ensino mais focados, além de direcionar as buscas sobre o tema.

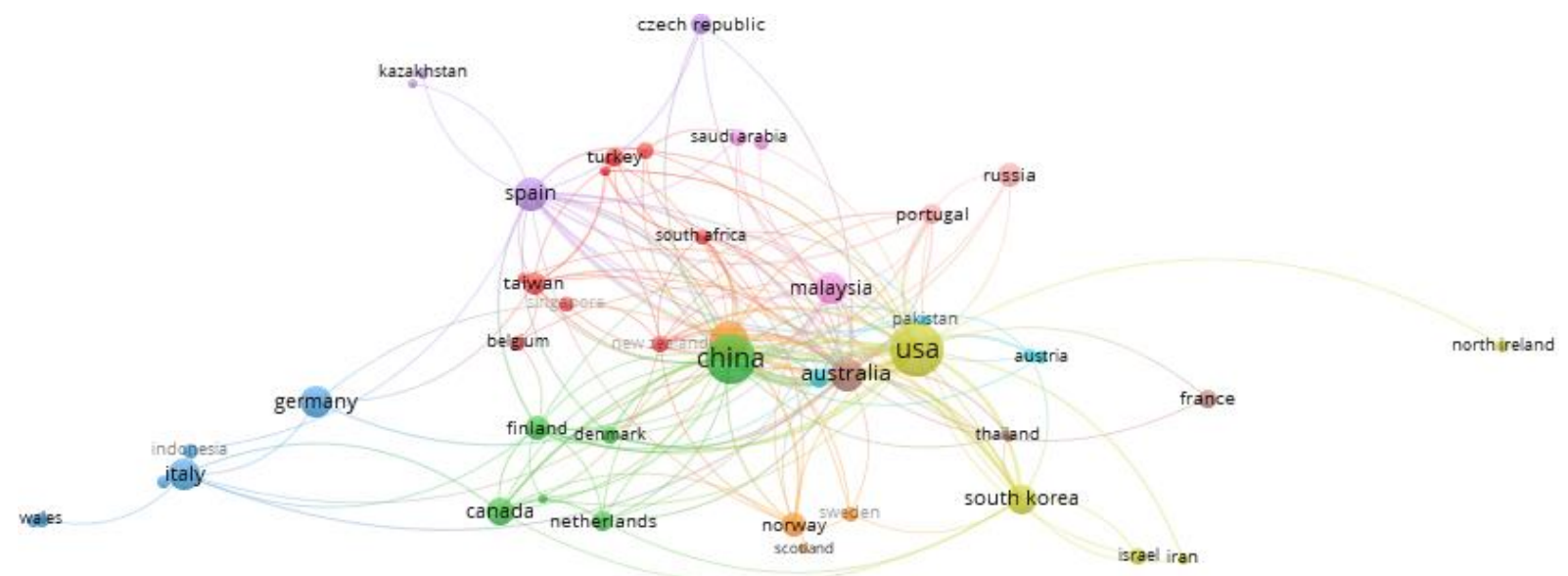

Figura 3: Citações - Países.

Tabela 2: Universidades relevantes

\begin{tabular}{lcc}
\hline Cluster & Universities & Weight citation \\
\hline $\mathbf{1}$ & Hong Kong Polytechnic University & 116 \\
$\mathbf{2}$ & Virginia Tech & 111 \\
$\mathbf{3}$ & University of Helsinki & 102 \\
$\mathbf{4}$ & Curtin University & 84 \\
$\mathbf{5}$ & Delft University of Technology & 52 \\
$\mathbf{6}$ & Chung-Ang University & 52 \\
$\mathbf{7}$ & California State University- Fresno & 44 \\
$\mathbf{8}$ & Penn State University & 40 \\
$\mathbf{9}$ & City University of Hong Kong & 39 \\
$\mathbf{1 0}$ & National University of Singapore & 28 \\
\hline
\end{tabular}


Tabela 3: Jornais relevantes

\begin{tabular}{lcc}
\hline Cluster & Journal & Weight citation \\
\hline $\mathbf{1}$ & Automation in construction & 434 \\
$\mathbf{2}$ & Journal of professional issues in engineering education and & 124 \\
$\mathbf{3}$ & practice & 90 \\
$\mathbf{4}$ & Engineering construction and architectural management & 71 \\
$\mathbf{5}$ & Journal of information technology in construction & 68 \\
$\mathbf{6}$ & International journal of engineering education & 39 \\
$\mathbf{7}$ & Construction management and economics & 38 \\
$\mathbf{8}$ & International journal of project management & 37 \\
$\mathbf{9}$ & Journal of civil engineering and management & 31 \\
$\mathbf{1 0}$ & Integrating data science, construction and sustainability & 23 \\
\hline
\end{tabular}

\section{Referências}

[1] J. Fridrich and K. Kubečka, "BIM - The Process of Modern Civil Engineering in Higher Education," Procedia - Soc. Behav. Sci., vol. 141, pp. 763-767, 2014.

[2] W. W. Hood and C. S. Wilson, "The literature of bibliometrics, scientometrics, and informetrics," Scientometrics, 2001.

[3] Thomsom Reuters, "Whitepaper Using Bibliometrics : A guide to evaluating research performance with citation data.," 2008.

[4] I. Zupic and T. Čater, "Bibliometric Methods in Management and Organization," Organ. Res. Methods, 2015.

[5] S. A. Morris and B. Van Der Veer Martens, "Mapping research specialties," Annual Review of Information Science and Technology. 2008.

[6] F. Schmidt, "Meta-analysis: A constantly evolving research integration tool," Organ. Res. Methods, 2008.

[7] E. Garfield, "Is citation analysis a legitimate evaluation tool?," Scientometrics, 1979.

[8] H. Small, "Visualizing science by citation mapping," J. Am. Soc. Inf. Sci., 1999.

[9] G. Holton, H. Chang, and E. Jurkowitz, "How a scientific discovery is made: A case history," Am. Sci., 1996.

[10] N. J. van Eck and L. Waltman, "Visualizing Bibliometric Networks," in Measuring Scholarly Impact, 2014.

[11] N. J. van Eck and L. Waltman, "Software survey: VOSviewer, a computer program for bibliometric mapping," Scientometrics, 2010.

[12] E. Garfield, "Citation indexes for science," Science (80-. )., 1955.

[13] K. Börner, C. Chen, and K. W. Boyack, "Visualizing knowledge domains," Annu. Rev. Inf. Sci. Technol., 2003. 
[14] H. Small, “A SCI-Map case study: Building a map of AIDS research,” Scientometrics, 1994.

[15] A. Skupin, "The world of geography: Visualizing a knowledge domain with cartographic means," Proc. Natl. Acad. Sci., vol. 101, no. suppl 1, pp. 5274-5278, Apr. 2004.

[16] N. J. Van Eck, L. Waltman, R. Dekker, and J. Van Den Berg, "A comparison of two techniques for bibliometric mapping: Multidimensional scaling and VOS," J. Am. Soc. Inf. Sci. Technol., 2010.

[17] L. Waltman, N. J. van Eck, and E. C. M. Noyons, "A unified approach to mapping and clustering of bibliometric networks," J. Informetr., 2010.

[18] M. Hu, "BIM-Enabled Pedagogy Approach: Using BIM as an Instructional Tool in Technology Courses," 2019.

[19] P. M. Bosch-Sijtsema, P. Gluch, and A. A. Sezer, "Professional development of the BIM actor role," Autom. Constr., vol. 97, pp. 44-51, 2019.

[20] T.-H. Wu, F. Wu, C.-J. Liang, Y.-F. Li, C.-M. Tseng, and S.-C. Kang, "A virtual reality tool for training in global engineering collaboration," Univers. ACCESS Inf. Soc., vol. 18, no. 2, SI, pp. 243-255, Jun. 2019.

[21] C. S. Park, Q. T. Le, A. Pedro, and C. R. Lim, "Interactive Building Anatomy Modeling for Experiential Building Construction Education," 2016.

[22] W. Wu, G. Mayo, T. L. McCuen, R. R. A. Issa, and D. K. Smith, "Building Information Modeling Body of Knowledge. I: Background, Framework, and Initial Development," 2018.

[23] E. Hjelseth, "Integrated Approaches for Implementing Building Information Modelling (Bim) in Engineering Education," Proc. 8Th Int. Conf. Eng. Bus. Educ., 2015.

[24] F. Zamora-Polo, A. Luque-Sendra, F. Aguayo-Gonzalez, and J. Sanchez-Martin, "Conceptual Framework for the Use of Building Information Modeling in Engineering Education," Int. J. Eng. Educ., vol. 35, no. 3, pp. 744-755, 2019.

[25] E. A. Paern, D. J. Edwards, and M. C. P. Sing, "Origins and probabilities of MEP and structural design clashes within a federated BIM model," Autom. Constr., vol. 85, pp. 209-219, Jan. 2018.

[26] V. Stojanovic, M. Trapp, R. Richter, and J. Döllner, “A service-Oriented approach for classifying 3D points clouds by example of office furniture classification," 2018.

[27]F. Patino-Cambeiro, G. Bastos, J. Armesto, and F. Patino-Barbeito, "Multidisciplinary Energy Assessment of Tertiary Buildings: Automated Geomatic Inspection, Building Information Modeling Reconstruction and Building Performance Simulation," ENERGIES, vol. 10, no. 7, Jul. 2017. 